\title{
Multiple-level lumbar spondylolysis and spondylolisthesis
}

\author{
Xinyu Liu, MD, Lianlei Wang, MS, Suomao Yuan, MD, Yonghao Tian, MD, Yanping Zheng, MD, \\ and Jianmin Li, MD
}

Department of Orthopedic Surgery, Qilu Hospital of Shandong University, Jinan, Shandong, China

\begin{abstract}
OBJECT Lumbar spondylolysis and isthmic spondylolisthesis occur most commonly at only one spinal level. The authors report on 13 cases of lumbar spondylolysis with spondylolisthesis at multiple levels.

METHODS During July 2007-March 2012, multiple-level spondylolysis associated with spondylolisthesis was diagnosed in 13 patients ( 10 male, 3 female) at Qilu Hospital of Shandong University. The mean patient age was $43.5 \pm$ 14.6 years. The duration of low-back pain was $11.7 \pm 5.1$ months. Spondylolysis occurred at L-2 in 2 patients, L-3 in 4 patients, L- 4 in all patients, and L-5 in 5 patients. Spondylolysis occurred at 3 spinal levels in 3 patients and at 2 levels in 10 patients. All patients had spondylolisthesis at 1 or 2 levels. Japanese Orthopaedic Association and visual analog scale scores were used to evaluate preoperative and postoperative neurological function and low-back pain. All patients underwent pedicle screw fixation and interbody fusion or direct pars interarticularis repair.
\end{abstract}

RESULTS Both low-back pain scores improved significantly after surgery $(p<0.05)$. Postoperative radiographs or CT scans showed satisfactory interbody fusion or pars interarticularis healing. No breakage, dislodging, or loosening of the pedicle screw hardware was observed for any patient.

CONCLUSIONS Multiple-level lumbar spondylolysis and spondylolisthesis occurred more often in men. Most multiplelevel lumbar spondylolysis occurred at 2 spinal levels and was associated with sports, trauma, or heavy labor. Multiplelevel lumbar spondylolysis occurred mostly at L3-5; associated spondylolisthesis usually occurred at L-4 and L-5, mostly at L-4. The treatment principle was the same as that for single-level spondylolisthesis.

http://thejns.org/doi/abs/10.3171/2014.10.SPINE14415

KEY WORDS lumbar spine; multiple-level; spondylolisthesis; spondylolysis

$\mathrm{A}$ MONG the general population, the estimated incidence of lumbar spondylolysis is $3 \%-10 \%$ and that of isthmic spondylolisthesis is $2.6 \%-4.4 \% .^{4}$ Lumbar spondylolysis and isthmic spondylolisthesis occur most commonly at a single spinal level, usually the unilateral or bilateral L-5 pars interarticularis. Only a few cases of multiple-level lumbar spondylolysis have been reported. ${ }^{1-3,5-11}$ Ravichandran ${ }^{8}$ calculated that only $1.48 \%$ of patients with back pain have multiple-level spondylolysis. Sakai et al. ${ }^{9}$ reviewed CT scans of 2000 persons without low-back pain and found lumbar spondylolysis in 117 $(5.9 \%)$ and multiple-level spondylolysis in $5(0.3 \%)$.

Even fewer reports of multiple-level spondylolysis associated with spondylolisthesis are found in the literature. ${ }^{1,3,11}$ We report 13 such cases, provide a literature review, and briefly describe clinical and radiological features and management alternatives for patients with this condition.

\section{Methods}

From July 2007 through March 2012, a total of 13 patients (10 male, 3 female) received a diagnosis of multiplelevel spondylolysis associated with spondylolisthesis; 9 of these patients performed heavy manual labor. Mean patient age $( \pm \mathrm{SD})$ was $43.5 \pm 14.6$ years, and duration of lowback pain $( \pm \mathrm{SD})$ was $11.7 \pm 5.1$ months. Before surgery, all patients underwent routine and dynamic radiography, CT, and MRI. Spondylolysis was located at 3 spinal levels for 3 patients and at 2 levels for 10 patients (Fig. 1). Spondylolysis was located at L-2 in 2 patients (bilateral), L-3 in 4

ABBREVIATIONS JOA = Japanese Orthopaedic Association; VAS = visual analog scale

SUBMITTED April 28, 2014. ACCEPTED October 21, 2014.

INCLUDE WHEN CITING Published online December 19, 2014; DOI: 10.3171/2014.10.SPINE14415.

DISCLOSURE The authors report no conflict of interest concerning the materials or methods used in this study or the findings specified in this paper. 


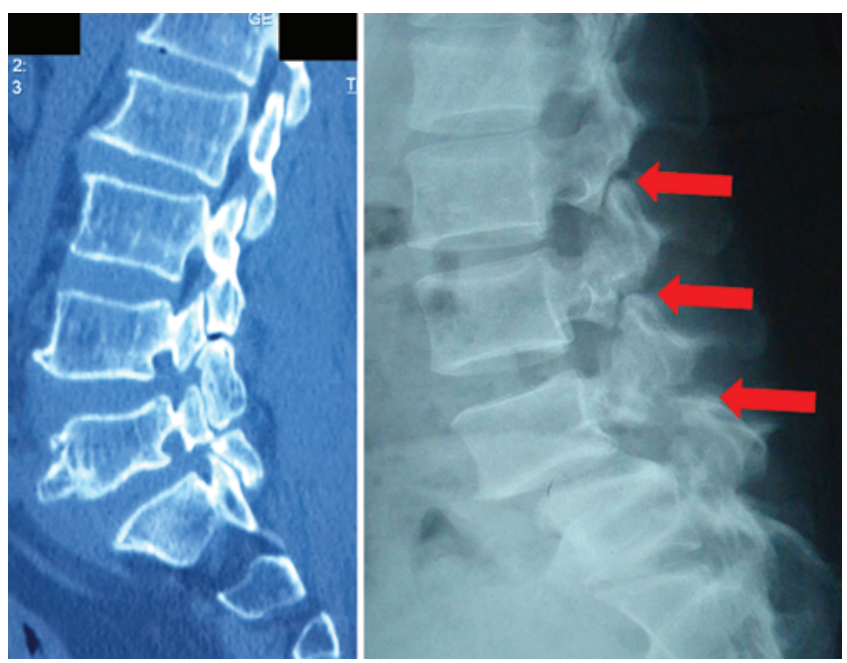

FIG. 1. Left: Two-level spondylolysis (L-4 and L-5) with spondylolisthesis (L4-5 and L5-S1). Right: Three-level spondylolysis (L-2, L-3, and $\mathrm{L}-4)$ (upper arrows) with spondylolisthesis (L4-5) (lower arrow). Figure is available in color online only.

patients (unilateral in 1 and bilateral in 3), L-4 in all patients (bilateral), and L-5 in 10 patients (bilateral in 7 and unilateral in 3). For 1 patient, a transitional vertebra (L-5) was observed. We used the Meyerding classification ${ }^{6}$ to evaluate the degree of spondylolisthesis. For all patients, spondylolisthesis occurred at L4-5 (Grade I for 11 patients and Grade II for 2 patients), for 6 patients at L5-S1 (Grade I for all 6 patients), and for 1 patient at L2-3 (Grade I). At spinal levels with spondylolisthesis or bilateral pars interarticularis defect with neurological deficit, posterior lumbar interbody fusion (11 levels) or transforaminal lumbar interbody fusion (9 levels) was performed. At spinal levels of pars interarticularis defects without instability or degenerative lumbar spinal canal stenosis, direct repair of spondylolytic defects was performed.

\section{Preoperative and Postoperative Assessments}

To assess neurological function, we used Japanese Orthopaedic Association (JOA) scores, and to assess lowback pain, we used a $10-\mathrm{cm}$ visual analog scale (VAS) (0 indicated no pain; 10 indicated the worst pain). The healing of pars interarticularis defects or interbody fusion was evaluated on plain radiographs or CT scans. The postoperative images were evaluated by 3 surgeons (Y.T., L.W., and J.L.), who did not know the patients' preoperative and postoperative status.

\section{Statistical Analyses}

SPSS version 12.0 software (SPSS, Inc.) was used for statistical analyses. In the event of statistical significance, a Student t-test was performed. The level of significance was set at $\mathrm{p}<0.05$. Mean values are presented \pm SD.

\section{Results}

The mean follow-up time was 30 months (range 25-36 months). VAS and JOA scores for low-back pain improved significantly after surgery $(\mathrm{p}<0.05)$. Preoperative JOA (maximum 29 points) and VAS scores were $15.7 \pm 2.0$ and $8.3 \pm 0.6$, respectively, and the latest postoperative JOA and VAS scores were $25.2 \pm 0.8$ and $1.8 \pm 0.8$, respectively. Plain radiographs and/or CT scans showed satisfactory interbody fusion or pars interarticularis healing. No breakage, dislodging, or loosening of the pedicle was documented in any patient.

\section{Illustrative Case}

A 56-year-old man with a 4-month history of low-back pain, bilateral leg pain, and numbness sought care at the Department of Orthopedic Surgery at Qilu Hospital. The pain had been caused by lifting heavy objects. After 4 months of conservative treatment including acupuncture, massage, bed rest, and nonsteroidal antiinflammatory drugs, the patient was admitted for surgery because of worsening back pain and leg numbness. His preoperative JOA and VAS scores for low-back pain were 8 and 10 , respectively. Radiographs, CT scans, and MR images showed bilateral spondylolytic defects at L-2, L-4, and L-5, associated with spondylolisthesis (retrolisthesis at L2-3 and anterolisthesis at L4-5) and spinal canal stenosis at L4-5. The patient underwent spinal canal decompression at L4-5, transforaminal lumbar interbody fusion at L2-3, posterior lumbar interbody fusion at L4-5 and L5-S1, and pedicle screw fixation at L2-S1 (Fig. 2).

\section{Discussion}

Similar to the etiology of single-level spondylolysis, the etiology of multiple-level lumbar spondylolysis is thought to be fatigue fractures..$^{10}$ Only a few reports of multiplelevel lumbar spondylolysis have been published..$^{1-3,5,7,8,10,11}$ The main data gleaned from the literature are shown in Table 1. Multiple-level lumbar spondylolysis occurs more often in men than in women.,10,11 Most cases are associated with sports, trauma, heavy labor, or Albers-Schönberg disease. ${ }^{3,7,10,11}$ Consistent with the trends reported in the literature, in our study most $(n=10)$ of the 13 patients were male, and 9 patients worked at jobs requiring heavy manual labor. Multiple-level lumbar spondylolysis occurs from L-1 to S-1, but according to the literature, it occurs mainly at L3-5, ${ }^{1-3,5,7,8,10,11}$ which is also consistent with the cases in our study. Moreover, 2-level spondylolysis is more common than 3- or 4-level spondylolysis. According to the literature, patients seek care for severe low-back pain or associated symptoms mainly in their teens and 20s (range 14-43 years). However, in our study, the mean age at which patients sought care was 43.5 years, which is much older than the age reported in the literature. This finding might be associated with the poor economic status of these patients, who mainly lived in rural areas of China. Their economic status might have prevented them from seeking medical treatment until severe symptoms (e.g., low-back pain, leg pain, and numbness) had arisen and might also be the reason why all spondylolisthesis was at 1 or 2 levels. According to the literature and our results, spondylolisthesis usually occurs at L-4 and L-5, mostly at L-4 (Fig. 1). This finding differs from reports of single- 

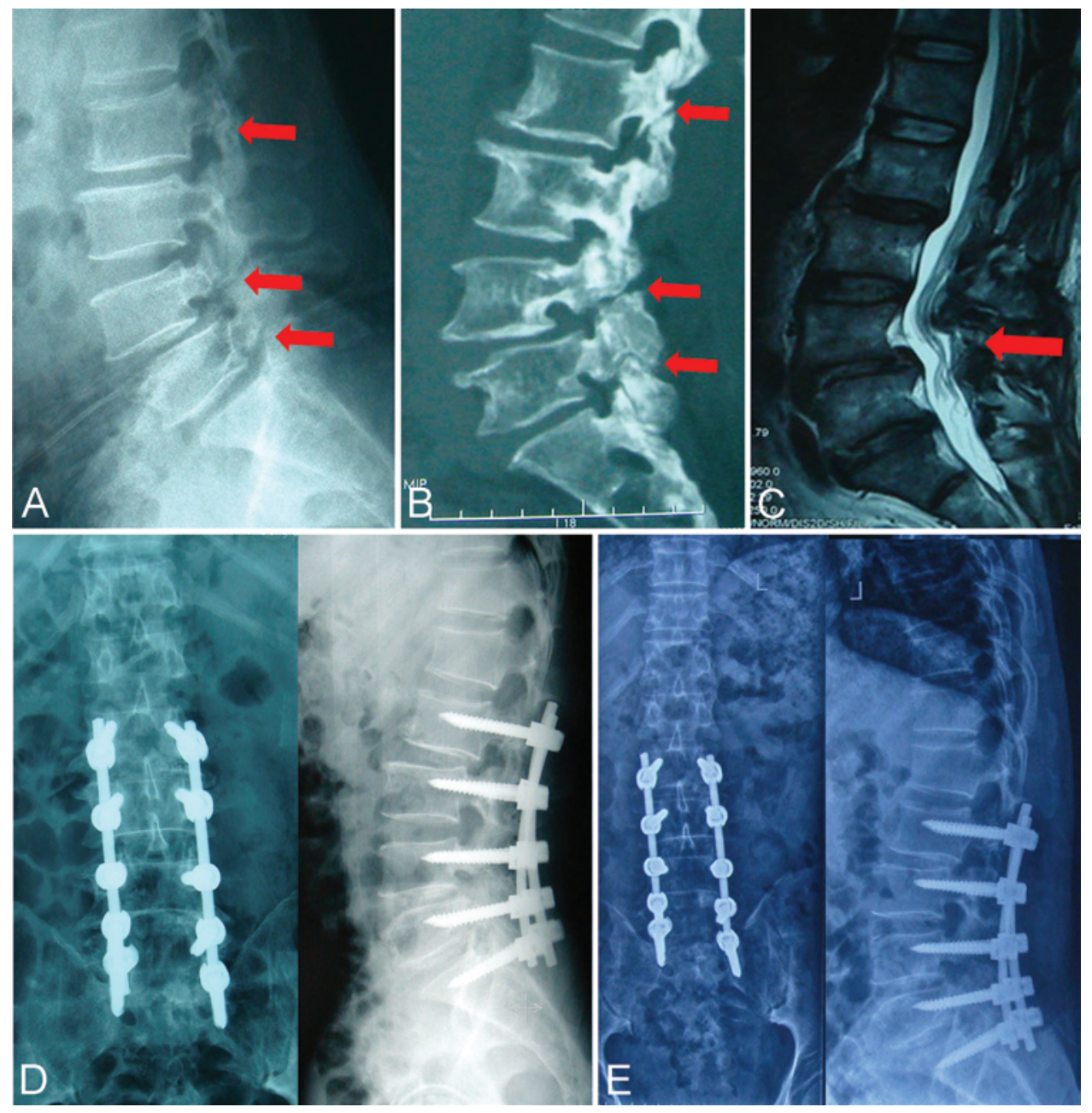

FIG. 2. Preoperative and postoperative images obtained in a 56-year-old man with a 4-month history of low-back pain, bilateral leg pain, and numbness. A-C: Spondylolysis at L-2, L-4, and L-5, and spondylolisthesis at L-4 (arrows). D: Postoperative radiographs. E: Two-year follow-up radiographs. Figure is available in color online only.

level lumbar spondylolysis and isthmic spondylolisthesis that most commonly occurs at L-5.

The main surgeries performed for spondylolysis and isthmic spondylolisthesis were direct repair of pars interarticularis defect, posterolateral fusion, posterior lumbar intervertebral fusion, and transforaminal lumbar intervertebral fusion. We thought that the principle of multiplelevel lumbar spondylolysis and spondylolisthesis treatment was the same as that for single-level spondylolisthesis. Multiple-level lumbar spondylolysis can be treated with conservative management or surgery. Conservative management is the standard treatment for spondylolysis and spondylolisthesis in cases in which the Meyerding grade is lower than III. ${ }^{3}$ Useful for pain relief are brace therapy, ${ }^{10}$ bed rest, lumbar flexion/distraction and activity modification, ${ }^{3}$ chiropractic manipulation, and rehabilitation of the spine $^{11}$ (Table 1). In general, surgery is necessary for patients who respond poorly to conservative treatment. ${ }^{2,7,10}$ For multiple-level lumbar spondylolysis without spondylolisthesis, pars interarticularis defects can be directly repaired using segmental wire fixation and bone grafting ${ }^{1}$ or using pedicle screw fixation, a laminar hook, and bone grafting. ${ }^{7}$ However, these methods cannot achieve adequate segmental stability for multiple-level lumbar spondylolysis with spondylolisthesis. In our study, all patients accepted pedicle screw fixation and underwent interbody fusion of most affected levels (12 levels in 6 patients). We performed direct repair of pars interarticularis defect only in relatively younger patients or in those who had a unilateral defect without degenerative disc changes and segmental instability. Direct repair of the pars interarticularis defect was performed at only 3 levels.

\section{Conclusions}

We have reported on 13 cases of multiple-level lumbar spondylolysis associated with spondylolisthesis and have summarized the results of a literature review. We found that multiple-level lumbar spondylolysis and spondylolisthesis occur more often in men than women and are associated with sports, trauma, or heavy manual labor. Multiple-level lumbar spondylolysis occurs mainly at L3-5, and spondylolisthesis usually occurs at L-4 and L-5, but mostly at L-4. 


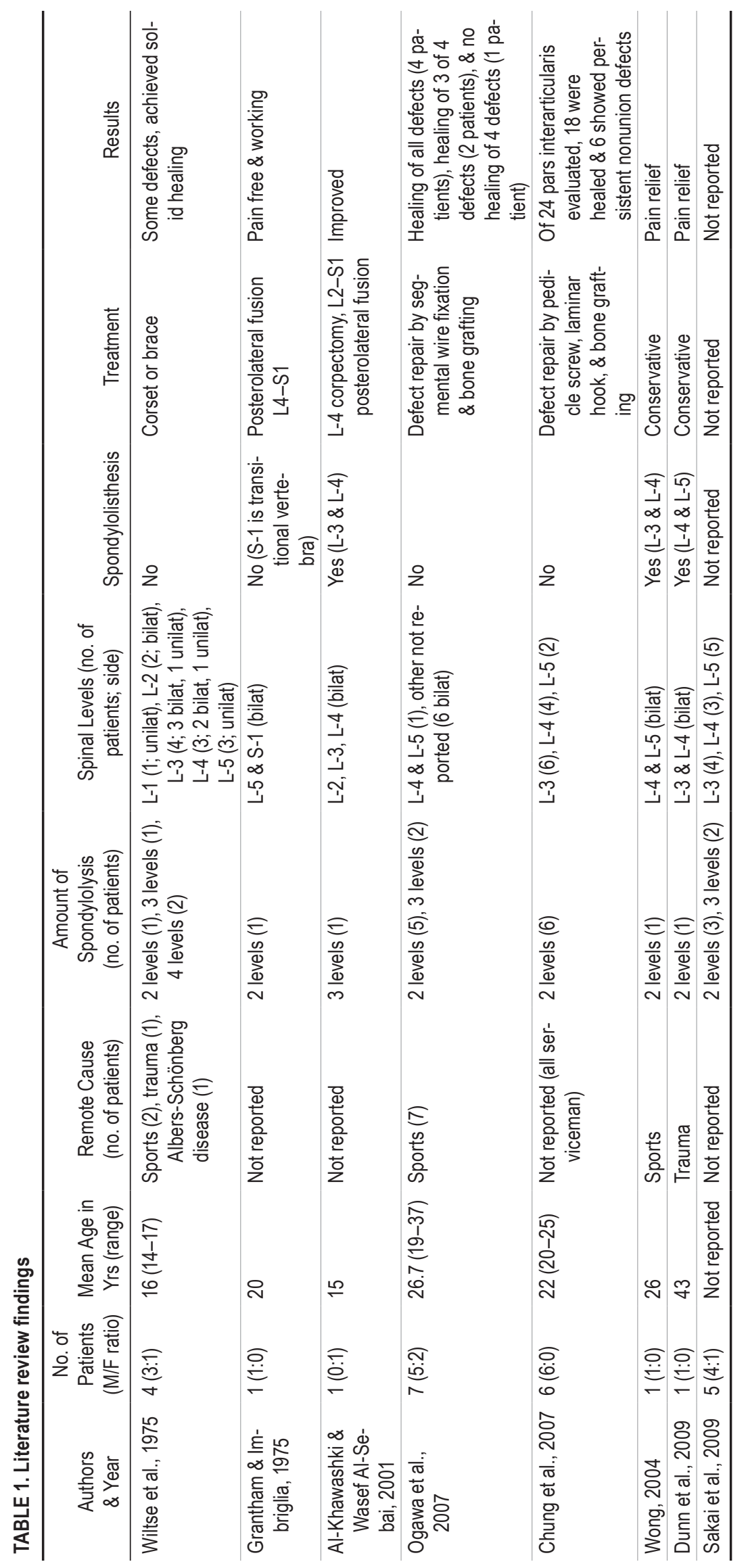




\section{References}

1. Al-Khawashki H, Wasef Al-Sebai M: Combined dysplastic and isthmic spondylolisthesis: possible etiology. Spine (Phila Pa 1976) 26:E542-E546, 2001

2. Chung CH, Chiu HM, Wang SJ, Hsu SY, Wei YS: Direct repair of multiple levels lumbar spondylolysis by pedicle screw laminar hook and bone grafting: clinical, CT, and MRIassessed study. J Spinal Disord Tech 20:399-402, 2007

3. Dunn AS, Baylis S, Ryan D: Chiropractic management of mechanical low back pain secondary to multiple-level lumbar spondylolysis with spondylolisthesis in a United States Marine Corps veteran: a case report. J Chiropr Med 8:125130, 2009

4. Ganju A: Isthmic spondylolisthesis. Neurosurg Focus 13(1):E1, 2002

5. Grantham SA, Imbriglia JE: Double-level spondylolysis and transitional vertebra. Case report. J Bone Joint Surg Am 57:713-714, 1975

6. Meyerding HW: Spondylolisthesis. Surg Gynecol Obstet 54:371-377, 1932

7. Ogawa H, Nishimoto H, Hosoe H, Suzuki N, Kanamori Y, Shimizu K: Clinical outcome after segmental wire fixation and bone grafting for repair of the defects in multiple level lumbar spondylolysis. J Spinal Disord Tech 20:521-525, 2007

8. Ravichandran G: Multiple lumbar spondylolyses. Spine (Phila Pa 1976) 5:552-557, 1980
9. Sakai T, Sairyo K, Takao S, Nishitani H, Yasui N: Incidence of lumbar spondylolysis in the general population in Japan based on multidetector computed tomography scans from two thousand subjects. Spine (Phila Pa 1976) 34:23462350, 2009

10. Wiltse LL, Widell EH Jr, Jackson DW: Fatigue fracture: the basic lesion is inthmic spondylolisthesis. J Bone Joint Surg Am 57:17-22, 1975

11. Wong LC: Rehabilitation of a patient with a rare multi-level isthmic spondylolisthesis: a case report. J Can Chiropr Assoc 48:142-151, 2004

\section{Author Contributions}

Conception and design: Liu. Acquisition of data: Liu, Tian. Analysis and interpretation of data: Wang. Drafting the article: Liu. Critically revising the article: Li. Reviewed submitted version of manuscript: Liu, Zheng, Li. Approved the final version of the manuscript on behalf of all authors: Liu. Statistical analysis: Yuan. Administrative/technical/material support: Li. Study supervision: Zheng.

\section{Correspondence}

Xinyu Liu, Department of Orthopedic Surgery, Qilu Hospital, Shandong University, 107 Wenhua Rd., Jinan, Shandong Province 250012, P. R. China. email: newyuliu@ hotmail.com. 